\title{
Control Strategies for Multilevel APFs Based on the Windowed-FFT and Resonant Controllers
}

\author{
Yang $\operatorname{Han}^{\dagger}$ \\ $\dot{\dagger}^{*}$ Dept. of Power Electronics, School of Mechatronics Engineering, University of Electronic Science and Technology \\ of China, Chengdu, China
}

\begin{abstract}
This paper presents control strategies for cascaded H-bridge multilevel active power filters (APFs). A current loop controller is implemented using a proportional-resonant (PR) regulator, which achieves zero steady-state error at target frequencies. The power balancing mechanism for the dc-link capacitor voltages is analyzed and a voltage balancing controller is presented. To mitigate the picket-fence effect of the conventional FFT algorithm under asynchronous sampling conditions, the Hanning Windowed-FFT algorithm is proposed for reference current generation (RCG). This calculates the frequency, amplitude and phase of individual harmonic components accurately and as a result, selective harmonic compensation (SHC) is achieved. Simulation and experimental results are presented, which verify the validity and effectiveness of the devised control algorithms.
\end{abstract}

Key words: Active power filter, Cascaded H-bridge, Hanning-window, Multi-level inverter, Power quality, Windowed-FFT

\section{INTRODUCTION}

Due to the proliferation of nonlinear loads, the harmonic pollution of the electric power systems is increasing rapidly. These nonlinear loads draw a large amount of distorted currents from utilities resulting in significant power quality contamination and severe electromagnetic interference in the nearby sensitive loads [1]. Active power filters (APFs) are considered to be the most effective solution for harmonic pollution problems, and they have been extensively studied in recent literature [2]-[4].

In [5], an active power filter and a static var compensator (SVC) with active powering capability was presented. It used a 27-level inverter, which was composed of three H-bridges connected to the same dc-link with their outputs connected through an output transformer. Multilevel and multi-cell voltage source inverters are increasingly being used for power quality conditioners [5]-[9]. In [6], a predictive control

Manuscript received Oct. 18, 2011; revised Mar. 23, 2012

Recommended for publication by Associate Editor Kyo-Beum Lee.

${ }^{\dagger}$ Corresponding Author: hanyang_facts@hotmail.com

Tel: +86-137-3060-6846, UESTC

*Dept. of Power Electronics, School of Mechatronics Engineering, University of Electronic Science and Technology of China, China with a flying capacitor balancing scheme was presented for multi-cell APFs in terms of redundant control states. In retrospect, the APFs are restricted to three-level inverters, since the complexity of controlling the capacitor voltages, while maintaining a high bandwidth, increases significantly as the number of levels increases [7].

On the other hand, APFs can be applied to medium -voltage (MV) applications using combined series active and parallel passive filters, or an active filter in series with parallel passive filters $[8,9]$. However, these solutions are not compact, especially when multiple passive filters are adopted. Without passive filters, there are two ways of applying an APF in the MV range, either by using a coupling transformer or by effectively extending device voltage ratings. However, the transformer approach shows the disadvantage of a large volume, a high cost, and a high bandwidth requirement to be in compatible with harmonic injection frequencies. The series operation of semiconductor switches faces the problems of unbalanced static and dynamic voltage sharing due to the spread of device characteristics and mismatched driver and snubber circuits [9], [10].

Among the various topologies for APFs, the cascaded H-bridge multilevel configuration is appreciated for high-power applications due to its modularity and flexibility in manufacturing [8]-[10]. However, it is restricted by the 


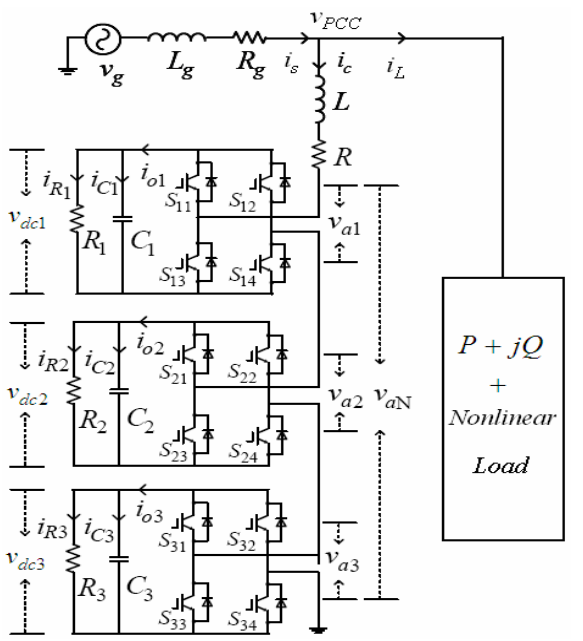

Fig. 1. Block diagram of the cascaded H-bridge (CHB) multilevel APF.

limited switching frequency of the power semiconductor devices, and achieving simultaneous dc capacitor voltage balancing and sufficient controller bandwidth is rather complicated. Systematic design guidelines for the CHB-APF have not been addressed in the previous studies. In this paper, an effective controller design methodology for the CHB-APF is proposed, including a proportional-resonant (PR) current controller, a dc-link voltage balancing controller (VBC) and the Windowed-FFT algorithm [11], [12] for reference current generation (RCG).

This paper is organized as follows. A brief introduction of cascaded H-bridge multilevel APFs is discussed in Section II. Control strategies for the CHB-APF are presented in Section III. A reference current generation (RCG) scheme based on the Hanning Windowed-FFT algorithm is illustrated in Section IV. Experimental results from a CHB-APF are presented in Section $\mathrm{V}$ and conclusions are given in Section VI.

\section{A BRIEF INTRODUCTION OF CASCADED H-BRIDGE MULTILEVEL APFS}

Fig. 1 shows a circuit diagram of a seven-level multilevel CHB-APF based on three H-bridge modules. In Fig. $1, L_{g}$ and $R_{g}$ indicate the line impedance. Each H-bridge includes four IGBT switches with anti-parallel diodes and a dc-link capacitor. Thus the output voltages of the CHB-APF can be derived as: $v_{a \mathrm{~N}}=v_{a 1}+v_{a 2}+v_{a 3}$.

Assuming $v_{d c 1}=v_{d c 2}=v_{d c 3}=V_{d c}$ in the steady state and the unipolar modulation scheme is adopted in the PWM process, each H-bridge will produce three voltage levels: $-V_{d c}, 0, V_{d c}$. With reference to the upper bridge, it is possible to set $v_{a 1}=+V_{d c}$ by turning on the switches $S_{11}$ and $S_{14}$ while it is possible to set $v_{a 1}=-V_{d c}$ by turning on the switches $S_{12}$ and $S_{13}$. Moreover, it is possible to set $v_{a 1}=0$ by turning on either $S_{11}$ and $S_{12}$ or $S_{13}$ and $S_{14}$. The lower bridges operate in a similar

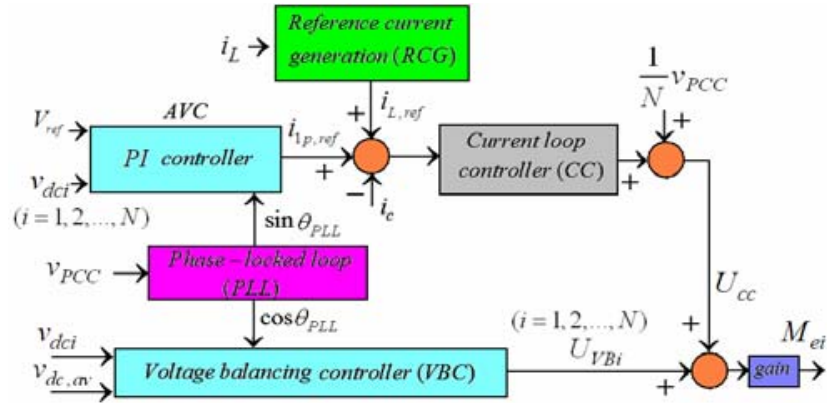

Fig. 2. Control strategies for the multilevel active power filter.

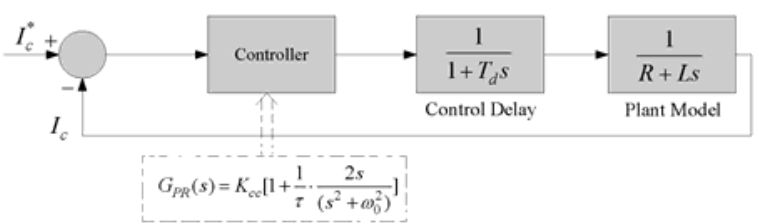

Fig. 3. Simplified block diagram for the current loop controller.

manner. Thus seven distinct voltage levels can be synthesized at the ac terminals [5]-[10].

\section{CONTROL StRATEGIES FOR A MULTILEVEL ACTIVE POWER FILTER}

Fig. 2 shows a control diagram of a cascaded H-bridge multilevel APF, which includes a phase-locked loop (PLL), a current loop controller, an average dc-link voltage controller (AVC), a dc-link voltage balancing controller (VBC), and the reference current generation (RCG) algorithm. The current controller and the voltage loop controller will be presented in this section and the RCG technique based on the Hanning Windowed-FFT algorithm will be presented in section IV.

\section{A. Resonant Current Controller Design}

The system equation across the coupling inductance can be derived as:

$$
v_{P C C}=v_{a N}+R \cdot i_{c}+L \cdot \frac{d i_{c}}{d t}
$$

A Applying the Laplace transformation (s-domain transform) to Eq.(1), assuming that $v_{d r}=v_{P C C}-v_{a N}$, the following is obtained:

$$
\frac{I_{c}(s)}{V_{d r}(s)}=\frac{1}{R+L \cdot s}
$$

Similar to the analysis adopted in $[3,4]$, the inner current loop is depicted in Fig. 3. The open-loop transfer function for the current loop controller is represented as:

$$
G_{\text {open }}(s)=G_{c c}(s) \cdot \frac{1}{1+T_{d} \cdot s} \cdot \frac{1}{R+L \cdot s}
$$

where $T_{d}$ denotes the control delay and $G_{c c}(s)$ represents the transfer function of the current controller, which can be a proportional controller, a proportional-integral (PI) controller, or a proportional-resonant controller. The closed-loop 


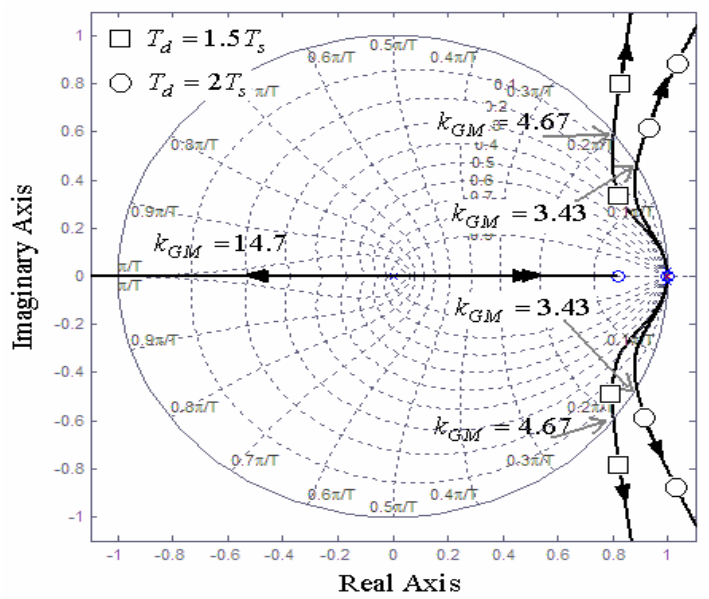

Fig. 4. Root locus analysis of the proportional-resonant (PR) controller.

transfer function for the current tracking control is denoted as:

$$
G_{\text {close }}(s)=\frac{G_{\text {open }}(s)}{1+G_{\text {open }}(s)}
$$

The proportional resonant (PR) current controller achieves perfect tracking performance for an alternating reference signal, which mimics the PI regulator implemented in the synchronous rotating reference frame with the following transfer function:

$$
G_{P R}(s)=K_{c c}\left[1+\frac{1}{\tau} \cdot \frac{2 s}{\left(s^{2}+\omega_{0}^{2}\right)}\right]
$$

where the parameter $\tau$ is defined as the time constant for the resonant controller and $\omega_{0}$ denotes the angular frequency of the fundamental grid voltage. Hence the PR regulator achieves zero steady-state error for the alternating signal at $\omega_{0}$ due to the infinite open-loop gain introduced by the PR regulator. Substituting Eq.(5) into the transfer function, Eq.(4), of the closed-loop current tracking scheme, the following is obtained:

$$
\begin{aligned}
G_{\text {close }}^{P R}(s)= & \frac{\tau K_{c c} s^{2}+2 K_{c c} s+\tau K_{c c} \omega_{0}^{2}}{\left\{\left(\tau T_{d} L\right) s^{4}+\left(T_{d} R+L\right) \tau s^{3}+\left[\left(R+K_{c c}\right) \tau+T_{d} L \omega_{0}\right] s^{2}\right.} \\
& \left.+\left[\left(T_{d} R+L\right) \tau \omega_{0}^{2}+2 K_{c c}\right] s+\tau \omega_{0}^{2}\left(R+K_{c c}\right)\right\}
\end{aligned}
$$

Fig. 4 shows a root locus plot of the closed-loop current tracking scheme using a PR regulator when the time constant is selected as $\tau=10 T_{s}$ ( $T_{s}$ is sampling time, $T_{s}=100 \mu \mathrm{s}$ ). It can be observed that the critical controller gains are 4.67 and 3.43, when $T_{d}=1.5 T_{s}$ and $T_{d}=2 T_{s}$, respectively.

\section{B. Dc-Link Voltage Balancing Control Algorithm}

It is well-know that the active and reactive power flow between the inverter and grid depend on the magnitude and phase of the synthesized multilevel voltage $v_{a N}$ with respect to

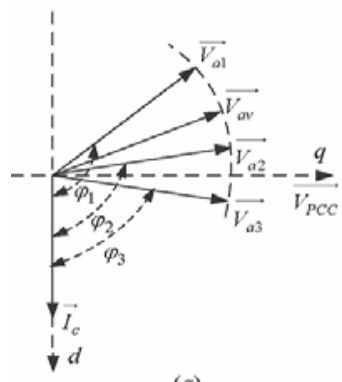

(a)

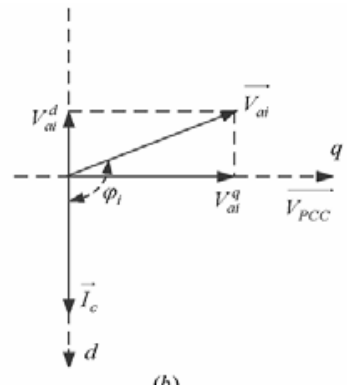

(b)
Fig. 5. Phasor representation of the individual H-bridge voltage with respect to its injection current.

the grid voltage $v_{P C C}$. The multilevel voltage $v_{a N}$ is the sum of the output voltage of the individual H-bridge modules (Fig. 1). The average output voltage for each inverter is denoted as:

$$
v_{a v}=\frac{1}{3} \sum_{i=1}^{3} v_{a i}=\frac{1}{3} v_{a N}
$$

Fig. 5 shows a phasor representation of the output voltage waveform of each H-bridge module. The phase displacement of the grid voltage $v_{P C C}$ and the reactive current $i_{c}$ are also illustrated. The active and reactive power delivered by the $i$ th H-bridge to the grid can be represented as:

$$
P_{i}=V_{a i} \cdot I_{c} \cdot \cos \varphi_{i}, Q_{i}=V_{a i} \cdot I_{c} \cdot \sin \varphi_{i}
$$

where $\varphi_{i}$ represents the phase angle between the multilevel APF current and the output voltage of each H-bridge module.

It can be seen in Fig. 5(a) that the active and reactive power delivered by each module is proportional to the in-phase and orthogonal components of its output voltage with respect to the current. Fig. 5(b) shows the fictitious $d-q$ reference frame which depicts the active and reactive components of the output voltage, denoted by the $d$ - and $q$-axis components, respectively. This is a projection of the individual output voltage to the two axes. Hence the active and reactive powers loaded by the $i$ th $\mathrm{H}$-bridge module can be derived by:

$$
P_{i}=V_{a i}^{d} \cdot I_{c}, Q_{i}=V_{a i}^{q} \cdot I_{c}
$$

In order to equally distribute the reactive power among each of the H-bridge modules, the $q$-axis component of the output voltage $v_{a i}(i=1,2,3)$ should be same. However, the active power absorption by each of the H-bridge modules may not be identical due to non-ideal dc-link parameters, such as IGBT or diode conduction losses, which are reflected by the unequal $d$-axis projection of the output voltage $v_{a i}$.

Therefore, the actual active power absorption by the $i$ th H-bridge module can be rewritten as:

$$
P_{i}=V_{a i}^{d} \cdot I_{c}=\left(V_{a v}^{d}+\Delta V_{a v}^{d}\right) \cdot I_{c}=P_{a v}+\Delta P_{i}
$$

where $P_{a v}$ represents the average active power absorption of the H-bridge modules, and $\Delta P_{i}$ represents the difference between the individual active power and the average active power absorption. In order to achieve an active power balance among the modules, the summation of the $d$-axis 


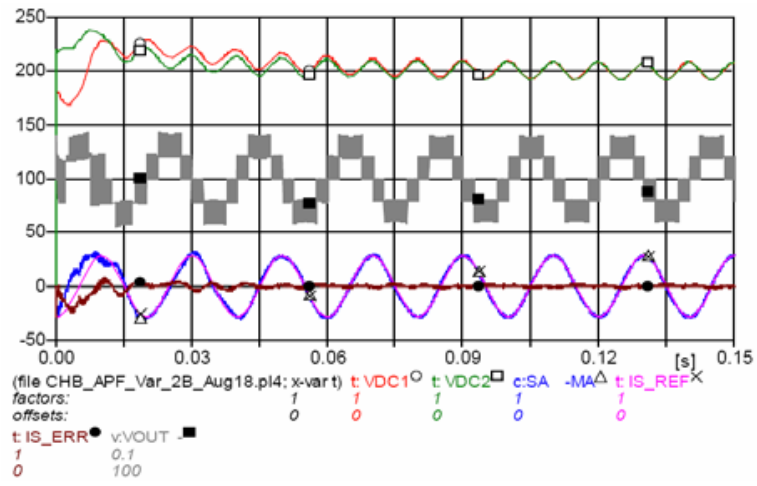

(a)

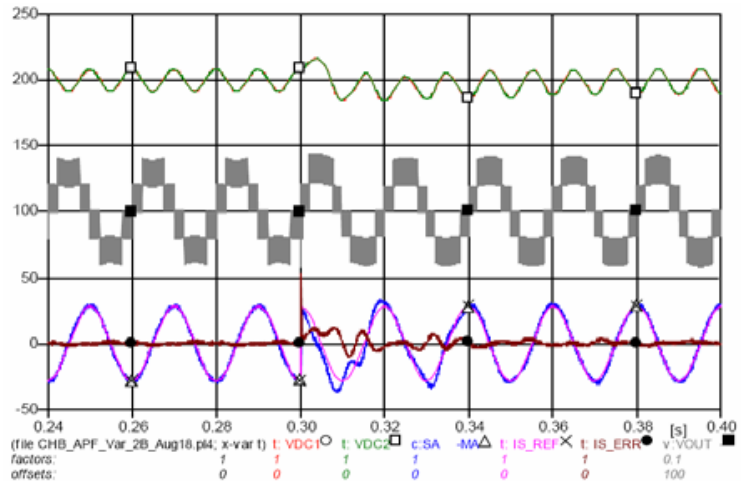

(b)

Fig. 6. The simulation results of the multilevel CHB-APF. (a) The convergence process after system start-up; (b) Transient response from the inductive mode to the capacitive mode.

projections of the output voltage of each of the H-bridge modules should be zero. By following this principle, the control scheme is devised as shown in Fig. 2.

This shows that the total active power absorption of the CHB-APF is balanced by regulating the average dc-link voltage using a proportional-integral (PI) controller, and that the output of the PI regulator is multiplied by a sine function synchronized with the grid using a software phase-locked loop (SPLL). The voltage balancing controller (VBC) is achieved by regulating the individual dc-link voltage with respect to the average dc-link voltage in order to regulate the active power distribution among the H-bridge modules. Proportional-integral regulators are also used and the outputs of the PI regulators are multiplied with a cosine function synchronized with the grid voltage. This projects the control error to the orthogonal axis with respect to the grid voltage [see Fig. 5]. After the voltage balancing signals are obtained, the total synthesized modulation signals can be derived as:

$$
M_{e i}=\frac{1}{v_{d c i}}\left(U_{V B i}+U_{c c}\right),(i=1,2,3)
$$

where $U_{c c}$ represents the output signal of the current loop controller.

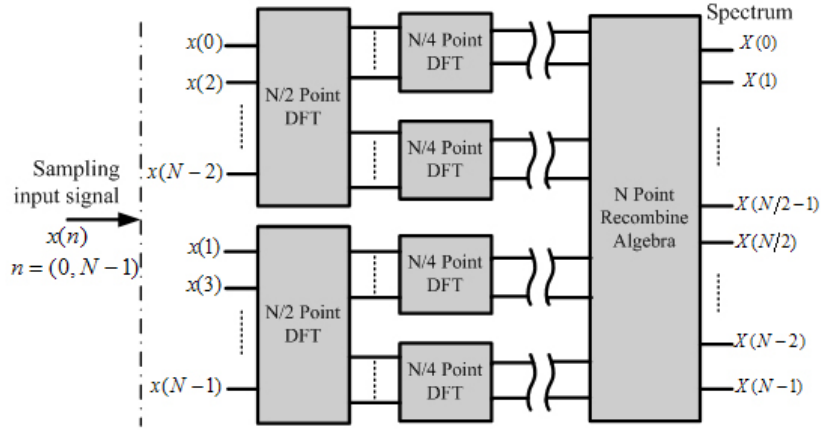

Fig. 7. Radix-2 decimation-in-time FFT.

To validate the voltage balancing control scheme, a digital simulation of the two-block CHB-APF using the Alternative Transient Program (ATP/EMTP) is conducted. The system parameters are: $L_{\mathrm{g}}=50 \mu \mathrm{H}, \quad R_{\mathrm{g}}=20 \mathrm{~m} \Omega, L=1.5 \mathrm{mH}$, and $R=50 \mathrm{~m} \Omega$. The dc-link parameters are: $C_{l}=C_{2}=2000 \mu \mathrm{F}$ and $R_{l}=R_{2}=39000 \Omega$. The sampling time $T_{s}=100 \mu \mathrm{s}$, the grid voltage $v_{s a}=220 \mathrm{~V}(\mathrm{rms})$, and the target dc voltage is $200 \mathrm{~V}$.

Fig. 6 shows the time domain simulation results of the CHB-APF for reactive compensation purposes, where the dc-link voltages, the synthesized multilevel voltage, the grid side current and its reference, as well as the tracking error are illustrated. The convergence process after system start-up is shown in Fig. 6(a), where the initial dc-link voltages are set to $180 \mathrm{~V}$ and $220 \mathrm{~V}$, respectively. It can be observed that the dc-link voltages converge to the same value after a few cycles. Fig. 6(b) shows the dynamic response of the CHB-APF when the reference current undergoes a transient change from the inductive mode to the capacitive mode.

\section{REFERENCE CURRENT GENERATION METHOD}

The existing approaches to compute APF reference current can be divided into the time-domain and the frequencydomain methods [11]. The time-domain approaches, such as the instantaneous p-q method, suffer from errors in case of grid distortions and PLL dynamics. The frequency-domain methods, on the other hand, utilize the discrete Fourier transform (DPF), or the recursive DFT algorithms [12], for the individual harmonic estimation of nonlinear currents. This section presents the Hanning-windowed FFT for reference current generation (RCG) for the CHB-APF.

\section{A. Review of the Fast Fourier Transform}

If $x(\mathrm{t})$ is a continuous periodical signal with a period $T$ and it satisfies the Dirichlet condition, it can be represented by the Fourier series [12]:

$$
x(t)=\sum_{k=-\infty}^{\infty} X\left(k \omega_{1}\right) e^{j k \omega_{1} t}
$$


TABLE I

COEFFICIENTS OF COSINE WINDOW

\begin{tabular}{cccccc}
\hline \hline Window & $K$ & $a_{0}$ & $a_{1}$ & $a_{2}$ & $a_{3}$ \\
\hline Rectangular & 0 & 1 & 0 & 0 & 0 \\
Hanning & 1 & 0.5 & 0.5 & 0 & 0 \\
Hamming & 1 & 0.54 & 0.46 & 0 & 0 \\
Blackman & 2 & 0.42 & 0.50 & 0.08 & 0 \\
Blackman-Harris & 3 & 0.3579 & 0.4883 & 0.1413 & 0.0117 \\
\hline \hline
\end{tabular}

where $\omega_{1}=2 \pi / T$ and $X\left(k \omega_{1}\right)$ is the Fourier coefficient at the $k$ th harmonic in the frequency domain. It can be observed from Eq.(12) that both the time and frequency domain signals have an infinite length. Assuming that an arbitrary electrical signal $x(\mathrm{t})$ is characterized by the fundamental frequency $f_{1}\left(f_{1}=1 / T\right)$ sampled with a rate of $N$ points per cycle, i.e., $T_{\mathrm{s}}=T / N$ and the sampling frequency $f_{\mathrm{s}}=1 / T_{\mathrm{s}}$ are as follows:

$$
x(n)=x(t) \mid t=\frac{n}{f_{s}}, n=0,1, \ldots, N-1
$$

Its FFT can be expressed as:

$$
X\left(\omega_{k}\right)=\sum_{n=0}^{N-1} x(n) e^{-j(2 \pi / N) n k}, k=0,1, \ldots, h
$$

where $\omega_{\mathrm{k}}=(2 \pi / T) k, X\left(\omega_{\mathrm{k}}\right)$ represents the spectrum of $x(n)$. The signal $x(n)$ is supposed to repeat itself for every $T$ interval, hence the angular frequency resolution of the spectrum is determined by the length of the signal:

$$
\Delta \omega=\omega_{1}=2 \pi / T
$$

The FFT algorithm uses an operation called decimation that relies on the recursive decomposition of an $N$-point DFT into two DFT transforms of N/2 points [see Fig.7]. The interpolated-FFT algorithm based on a cosine window (CW) is presented in the next section.

\section{B. Interpolation Algorithm based on a Cosine Window}

The generalized expression for the $K$-term cosine window is represented as [12]:

$$
w_{K}(n)=\sum_{k=0}^{K}(-1)^{k} a_{k} \cos \left(\frac{2 \pi}{N} k n\right), n=0,1, \ldots, N-1
$$

where $a_{k}(k=0$ to $K)$ are the window coefficients and $N$ is the number of acquired samples. In order to use the FFT directly, $N$ should be the power of two ( $N=2^{m}$, where $m$ is an integer). To meet the requirement of interpolation, the following equation should be satisfied for the coefficients:

$$
\sum_{k=0}^{K} a_{k}=1, \sum_{k=0}^{K}(-1)^{k} a_{k}=0
$$

where $a_{0}=\frac{C_{2 K-2}^{K-1}}{2^{2 K-2}}, a_{k}=\frac{C_{2 K-2}^{K-k-1}}{2^{2 K-3}}, k=1,2 . . K, C_{m}^{p}=\frac{m !}{(m-p) ! p !}$.

The values of $K$ and $a_{k}$ determine the various window functions, and the corresponding coefficients of the popular cosine windows are listed in Table I.

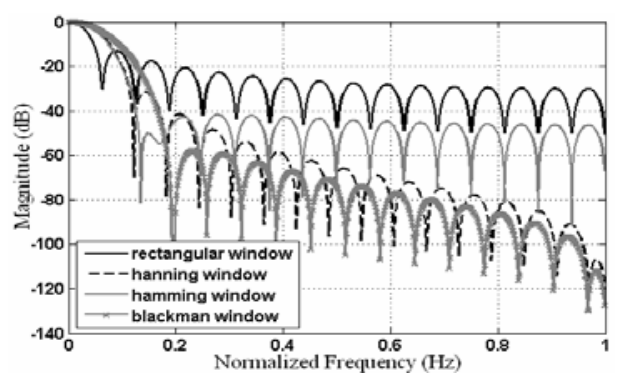

Fig. 8. Comparison of different K-term cosine window function spectrums.

The FFT of the $K$-term cosine window $w_{K}(n)$ is given by:

$$
\begin{aligned}
& W(\lambda)=\sin (\pi \lambda) e^{-j \pi \lambda} e^{j \pi \lambda / N} \\
& \times \sum_{k=0}^{K-1}(-1)^{k} 0.5 a_{k}\left[\frac{e^{-j \pi k / N}}{\sin \frac{\pi}{N}(\lambda-k)}+\frac{e^{j \pi k / N}}{\sin \frac{\pi}{N}(\lambda+k)}\right], \\
& \lambda=0,1, \ldots, N-1
\end{aligned}
$$

When $N>>1$, the FFT of the $K$-term maximum side lobe decay window can be approximated by:

$$
W(\lambda)=\frac{N \sin (\pi \lambda)}{2^{2 K-2} \pi \lambda} e^{-j \pi \lambda} e^{j \frac{\pi \lambda}{N}} \frac{(2 K-2) !}{\prod_{k=1}^{K-1}\left(k^{2}-\lambda^{2}\right)}
$$

The leakage effect can be analyzed from the spectra of the window functions, as denoted in Eq.(17). Fig.8 shows the spectra of the different window functions. Generally, a multi-frequency signal can be represented as:

$$
x(n)=\sum_{m=1}^{M} A_{m} \cos \left(2 \pi f_{m} n / f_{s}+\varphi_{m}\right), n=0,1, . ., N-1
$$

where $A_{m}, f_{m}$ and $\varphi_{m}$ are the amplitude, frequency and phase of the $m$ th harmonic component respectively, and $N$ is the number of acquired samples.

It should be noted that the sampling process is considered in the non-coherent sampling mode, and that the spectrum of $x(n)$ is affected by the leakage errors. Therefore, $x(n)$ is multiplied by a suitable window sequence $w(n)$. Thus, the FFT of the resulting signal $x_{\mathrm{w}}(n)=x(n) . w(\mathrm{n})$ is given by:

$$
\begin{aligned}
& X_{w}(\lambda)=\sum_{m=1}^{M} \frac{A_{m}}{2 j} W\left(\lambda-\lambda_{m}\right) e^{j \varphi_{m}} \\
& -\sum_{m=1}^{M} \frac{A_{m}}{2 j} W\left(\lambda+\lambda_{m}\right) e^{-j \varphi_{m}}, \lambda \in[0, N)
\end{aligned}
$$

where $\lambda$ represents the normalized frequency in the spectral line, $W(\lambda)$ is the FFT of $w(n)$ and $\lambda_{\mathrm{m}}=f_{\mathrm{m}} / f_{0}$ with $f_{0}=f_{\mathrm{s}} / N$. The second term in Eq.(21) represents the image part of the spectrum. Thus, $X_{w}(\lambda)$ can be written as:

$$
X_{w}(\lambda) \cong \frac{A_{m}}{2 j} W\left(\lambda-\lambda_{m}\right) e^{j \varphi_{m}}, \lambda \cong \lambda_{m}, m=1,2, \ldots, M
$$

The relationship between the frequencies $f_{\mathrm{m}}$ and $f_{\mathrm{s}}$ is: 


$$
\frac{f_{m}}{f_{s}}=\frac{\lambda_{m}}{N}=\frac{l_{m}+\delta_{m}}{N}, m=1,2, \ldots, M
$$

where $l_{\mathrm{m}}$ and $\delta_{\mathrm{m}}$ are the integer part and the fractional part of $\lambda_{\mathrm{m}}\left(-0.5 \leq \delta_{\mathrm{m}} \leq 0.5\right)$. Generally, $l_{\mathrm{m}}$ can be easily obtained as:

$$
l_{m}=\operatorname{round}\left(N f_{m} / f_{s}\right)
$$

If the sampling process is synchronized, $\delta_{m}=0$. However, strictly synchronized sampling is difficult to realize for most practical applications, which implies that $\delta_{m} \neq 0$. Thus the windowed interpolation approach based on the FFT is used to estimate $\delta_{\mathrm{m}}$, the following parameter $\alpha_{\mathrm{m}}$ is evaluated as:

$$
\alpha_{m}=\left\{\begin{array}{l}
\frac{\left|X_{w}\left(l_{m}\right)\right|}{\left|X_{w}\left(l_{m}-1\right)\right|},-0.5 \leq \delta_{m}<0 \\
\frac{\left|X_{w}\left(l_{m}+1\right)\right|}{\left|X_{w}\left(l_{m}\right)\right|}, 0 \leq \delta_{m}<0.5
\end{array}\right.
$$

Equation (25) implies that $\left|X_{w}\left(l_{m}-1\right)\right| \geq\left|X_{w}\left(l_{m}+1\right)\right|$ when $-0.5 \leq \delta_{m}<0$ and that $\left|X_{w}\left(l_{m}-1\right)\right|<\left|X_{w}\left(l_{m}+1\right)\right|$ when $0 \leq \delta_{m}<0.5$. It also implies that the reciprocal holds true. From Eqs.(22) and (25), the parameter $\alpha_{m}$ becomes:

$$
\alpha_{m} \cong\left\{\begin{array}{l}
\frac{\left|W\left(\delta_{m}\right)\right|}{\left|W\left(1+\delta_{m}\right)\right|},-0.5 \leq \delta_{m}<0 \\
\frac{\left|W\left(1-\delta_{m}\right)\right|}{\left|W\left(\delta_{m}\right)\right|}, 0 \leq \delta_{m}<0.5
\end{array}\right.
$$

Combining Eq.(19) with Eq.(26), we get:

$$
\alpha_{m} \cong\left\{\begin{array}{l}
\frac{K+\delta_{m}}{K-1-\delta_{m}},-0.5 \leq \delta_{m}<0 \\
\frac{K-1+\delta_{m}}{K-\delta_{m}}, 0 \leq \delta_{m}<0.5
\end{array}\right.
$$

Then the parameter $\delta_{m}$ can be derived as:

$$
\delta_{m}=\left\{\begin{array}{l}
\frac{(K-1) \alpha_{m}-K}{\alpha_{m}+1},-0.5 \leq \delta_{m}<0 \\
\frac{K \alpha_{m}-K+1}{\alpha_{m}+1}, 0 \leq \delta_{m}<0.5
\end{array}\right.
$$

Therefore, the frequency $f_{m}$ can be estimated:

$$
\widehat{f_{m}}=\left\{\begin{array}{c}
\left(l_{m}+\frac{(K-1) \alpha_{m}-K}{\alpha_{m}+1}\right) \cdot \frac{f_{s}}{N},-0.5 \leq \delta_{m}<0 \\
\left(l_{m}+\frac{K \alpha_{m}-K+1}{\alpha_{m}+1}\right) \cdot \frac{f_{s}}{N}, 0 \leq \delta_{m}<0.5
\end{array}\right.
$$

Combining Eqs.(23) and (29), the amplitude $A_{m}$ can also be estimated:

$$
\widehat{A_{m}}=\frac{2^{2 K-1} \pi \delta_{m}\left|X_{w}\left(l_{m}\right)\right|}{N \sin \left(\pi \delta_{m}\right)(2 K-2) !} \prod_{k=1}^{K-1}\left(k^{2}-\delta_{m}^{2}\right)
$$

Similarly, the phase angle of the $m$ th order component can be obtained:

$$
\begin{aligned}
& \widehat{\varphi_{m}}=\operatorname{Phase}\left\{X_{w}\left(l_{m}\right)\right\}-\pi \delta_{m}\left(1-\frac{1}{N}\right) \\
& -\frac{\pi}{2} \operatorname{sign}\left(\delta_{m}\right)-\operatorname{Phase}\left\{W_{0}\left(-\delta_{m}\right)\right\}
\end{aligned},
$$

$$
\begin{aligned}
& W_{0}(\lambda)=\sum_{k=0}^{K-1}(-1)^{k} \frac{1}{2} a_{k}\left[\frac{e^{-j \pi k / N}}{\sin \frac{\pi}{N}(\lambda-k)}+\frac{e^{j \pi k / N}}{\sin \frac{\pi}{N}(\lambda+k)}\right], \\
& \text { and } \operatorname{sign}\left(\delta_{m}\right)=\left\{\begin{array}{c}
-1,-0.5 \leq \delta_{m}<0 \\
1,0 \leq \delta_{m}<0.5
\end{array} .\right.
\end{aligned}
$$

The general expression for calculating the frequency, amplitude and phase of the signal by adopting the $K$-term cosine window interpolation. As for the rectangular window, the spectrum of the windowed signal at the integral points is:

$$
X_{w}(\lambda)=X(\lambda), \lambda=0,1, \ldots, N-1
$$

As for the Hanning window, $X_{w}(\lambda)$ is:

$$
X_{w}(\lambda)=\left\{\begin{array}{c}
0.5 X(\lambda)-0.25 X(\lambda+1), \lambda=0, N-1 \\
0.5 X(\lambda)-0.25[X(\lambda-1)+X(\lambda+1)], \lambda \in[1, N-2]
\end{array}\right.
$$

where $\lambda$ represents the normalized frequency expressed in bin, according to IEC standard 61000-4-7 and 61000-4-30 for the measurement of harmonics and inter-harmonics [13]. Assuming that the signal is given as shown in Eq.(20), the following expression can be obtained from Eqs.(23) and (24):

$$
\lambda_{m}=\Delta f \times m / f_{0}, l_{m}=\operatorname{round}\left(\Delta f \times m / f_{0}\right)
$$

where $f_{0}=f_{\mathrm{s}} / N$. If $\Delta f=5 \mathrm{~Hz}, m$ is the integral multiple of 10 (the fundamental frequency $f_{1}$ is assumed to be $50 \mathrm{~Hz}$ ), which implies that $f_{\mathrm{m}}$ is the mth harmonic frequency. In other words, $l_{10}, l_{20}, \ldots, l_{10 n}$ represents the spectral line of the harmonic component. Meanwhile, $l_{10+\mathrm{i}}, l_{20+\mathrm{i}}, \ldots, l_{10 \mathrm{n}+\mathrm{i}}(i=1,2,3 \ldots, 9)$ represents the spectral line of the interharmonic component.

However, since the strictly synchronized sampling cannot be satisfied, the parameters of the $f_{m}$ component are obtained according to the adjacent two spectral lines, namely, the $l_{m}$ th and $\left(l_{m}+1\right)$ th or the $l_{m}$ th and $\left(l_{m}-1\right)$ th lines. The coefficient $\alpha_{m}$ is the same as that expressed in Eq.(25). As for the rectangular window, the coefficient $\delta_{m}$ is given as:

$$
\delta_{m}=\frac{1}{1+\alpha_{m}}
$$

Then, the frequency, amplitude, phase can be derived as:

$$
\begin{gathered}
\widehat{f_{m}}=\left(l_{m}-\delta_{m}\right) \times f_{s} / N \\
\widehat{A_{m}}=\frac{2 \pi}{N} \delta_{m} \frac{\left|X_{w}\left(l_{m}\right)\right|}{\sin \left(\pi \delta_{m}\right)} \\
\widehat{\varphi_{m}}=\operatorname{Phase}\left(l_{m}\right)+\pi \delta_{m}
\end{gathered}
$$

where $\operatorname{Phase}\left(l_{m}\right)=\operatorname{atan}\left(\operatorname{Imag}\left(X_{\omega}\left(l_{m}\right)\right) / \operatorname{Real}\left(X_{\omega}\left(l_{m}\right)\right)\right)$.

As for the Hanning window, the coefficient $\delta_{\mathrm{m}}$ and amplitude $A_{m}$ are described as:

$$
\begin{gathered}
\delta_{m}=\frac{2 \alpha_{m}-1}{1+\alpha_{m}} \\
\widehat{A_{m}}=\frac{2 \pi}{N} \delta_{m}\left(1-\delta_{m}^{2}\right) \frac{\left|X_{w}\left(l_{m}\right)\right|}{\sin \left(\pi \delta_{m}\right)}
\end{gathered}
$$

The expressions for the frequency and phase angles are the same as those in Eqs.(37) and (39). Therefore when $m=10 n$ 


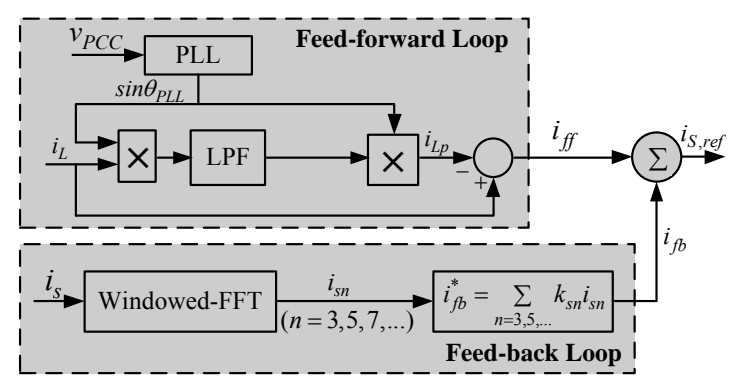

Fig. 9. The block diagram for the reference current generation algorithm.

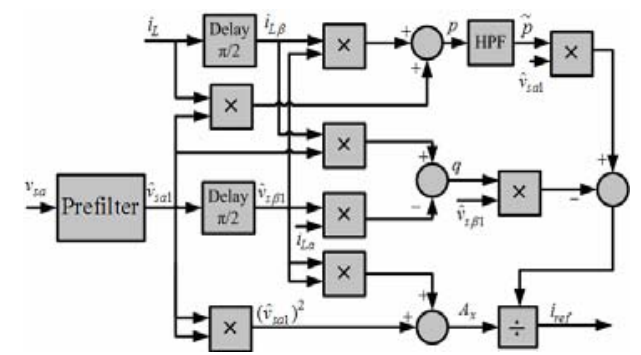

Fig. 10. Diagram of the single-phase IRPT-based RCG scheme [13].

$(n=1,2 . ., h)$, the amplitude, frequency and phase of the $n$th harmonic can be derived From Eqs.(37)-(41).

\section{The Reference Current Generation Scheme for the CHB-APF based on the Windowed-FFT Algorithm}

The Hanning window-based interpolation has increased the precision with a high accuracy in frequency, amplitude and phase angle estimation. The obtained frequency, amplitude and phase angle are utilized to reconstruct the reference signal for the CHB-APF [see Fig.9]. The grid current is processed by using the windowed-FFT and the target harmonic components are selected and multiplied by the control gains and as a result, the feedback signal $i_{f b}$ is obtained. The feed-forward loop in Fig.9 is based on the low pass filter approach, which subtracts the fundamental active component from the load current.

\section{Comparison with the IRPT-based RGC-scheme}

The instantaneous reactive power theory (IRPT) or $p-q$ theory is one of most popular algorithms for extracting the reference for active power filters [13]. The single-phase $p-q$ theory is based on an instantaneous $\pi / 2$ lag of the voltage and current to define the original system as a pseudo two-phase system. As shown in Fig.10, the original source voltage and load current are considered as quantities on the $\alpha$ - and $\beta$-axis. These quantities are obtained by a $\pi / 2$ lag of the source voltage and the load current. The reference current is:

$$
i_{\text {ref }}=\frac{1}{A_{x}} \cdot\left(\tilde{p} \hat{v}_{s a 1}-q \hat{v}_{s \beta 1}\right),
$$

where the parameters $A_{x}, p$ and $q$ are described as:
$A_{x}=\left(\hat{v}_{s a 1}\right)^{2}+\left(\hat{v}_{s \beta 1}\right)^{2}, p=i_{L a} \hat{v}_{s a 1}+i_{L \beta} \hat{v}_{s \beta 1}, q=i_{L \beta} \hat{v}_{s a 1}-i_{L a} \hat{v}_{s \beta 1}$.

\section{EXPERIMENTAL RESULTS}

To verify the validity and effectiveness of the proposed multilevel CHB-APF and its control strategies, a prototype system is built, based on three H-bridge modules. The proposed multilevel CHB-APF is implemented using a digital signal processor (DSP) from Texas Instrument, where the sampled dc-link voltages, the grid voltage and the inverter current are processed using the devised control algorithm.

The DSP is responsible for the initialization, A/D sampling, over-current/voltage protection, dc-link voltage balancing control as well as the current loop tracking control algorithms. The obtained modulation signals, denoted by $M_{e 1}, M_{e 2}$ and $M_{e 3}$ in Fig. 2, are sent to a field programmable gate array (FPGA), which is utilized to generate phase-shifted carriers of $2.5 \mathrm{kHz}$. These carriers are compared with the modulation signal to generate switching signals for each of the H-bridge modules. The controller parameters are listed in Table II.

Fig. 11 shows the reactive compensation mode of the CHB-APF. The rms value of the dc-link voltages (ch-1, ch-2) are about $45 \mathrm{~V}$ and the waveforms show perfect matching with each other. The rms values of the output voltages $v_{a 1}$ and $v_{a 2}$ (ch-3, ch-4) are about 35V. In Fig. 11(a), the grid current (ch-6) is leading the multilevel voltage $v_{a N}$ (ch-5) by 90 degrees, indicating that the CHB-APF is injecting capacitive reactive power into the grid. In Fig. 11(b), the grid current (ch-6) is lagging the multilevel voltage $v_{a N}$ (ch-5) by 90 degrees, indicating that the CHB-APF is absorbing capacitive reactive power from the grid.

Fig. 12 shows the dynamic response of the three-module CHB-APF for the compensation of a diode rectifier load. Under this case, the dc-link voltage and the grid side voltage are set as $20 \mathrm{~V}$ and $35 \mathrm{~V}$, respectively. The waveforms from ch-1 to ch-3 indicate dc-link voltages, while the waveforms from ch-4 to ch-6 indicate the source current, the APF current and the load current, respectively. Notably, the load current generated by the diode rectifier is a typical nonlinear current which contains the characteristic harmonic components of the orders $3,5,7,9$, etc.

Fig. 12(a) shows the results using the windowed-FFT based RCG scheme. It shows that the grid current is free of harmonics and that the unity power factor (UPF) is achieved at the grid side. The steady state voltage ripple across the dc-link capacitors is about $5 \mathrm{~V}$. Further, satisfactory dynamic performance is observed when the load is abruptly increased by adding a resistive load to the dc side of the diode rectifier. During the transient process, a sinusoidal waveform is achieved in the grid current and the stability of the dc-link capacitor voltage of the cascaded inverters is also guaranteed, with a transient voltage dip of $5 \mathrm{~V}$ in the dc-link voltages. Fig. 12(b) shows the results using the single-phase IRPT- based 


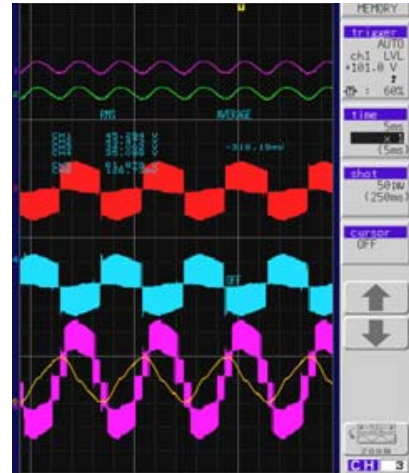

(a)

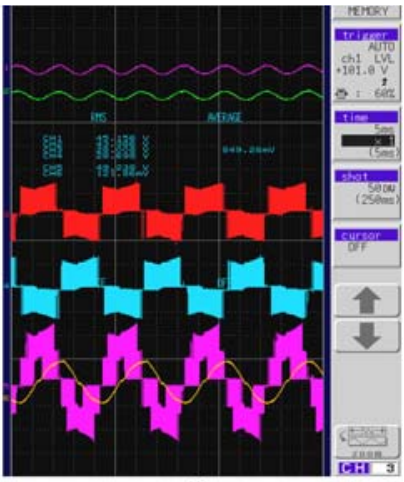

(b)

Fig. 11. The reactive compensation mode of the multilevel CHB-APF. (a) the capacitive operation mode. (b) the inductive operation mode.

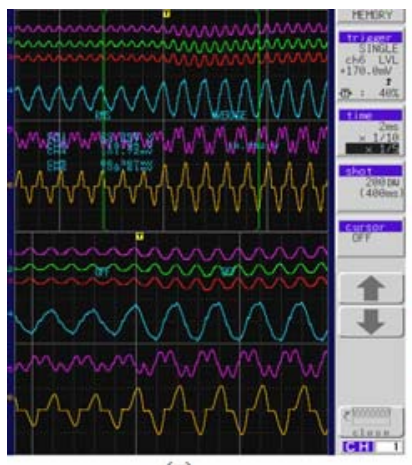

(a)

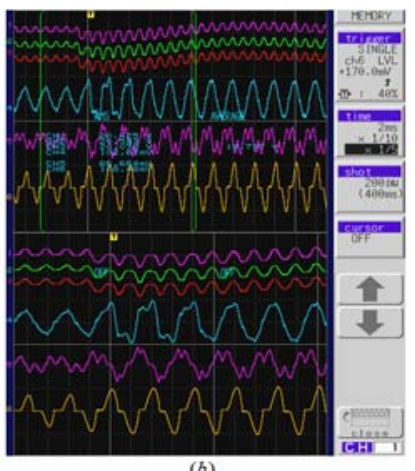

(b)
Fig. 12. The experimental result of the multilevel CHB-APF for nonlinear load compensation. (a) using the windowed-FFT-based RCG scheme. (b) using the single-phase IRPT-based RCG scheme.

TABLE II

The Controller Parameters for the Prototype Multilevel CHB-APF

\begin{tabular}{ll}
\hline \hline Proportional gain of PR current regulator $\left(K_{p c c}\right)$ & 2.5 \\
Time constant of PR current regulator $\left(\tau_{c c}\right)$ & $10 T_{s}$ \\
Proportional gain of average dc-voltage controller $\left(K_{p d c 1}\right)$ & 0.6 \\
Time constant of average dc-voltage controller $\left(\tau_{d c 1}\right)$ & $1000 T_{s}$ \\
Proportional gain of voltage-balancing controller $\left(K_{p d c 2}\right)$ & 0.2 \\
Time constant of voltage-balancing controller $\left(\tau_{d c 2}\right)$ & $2000 T_{s}$ \\
\hline \hline
\end{tabular}

RCG scheme, where the cutoff frequency of the high-pass filter (HPF) is $10 \mathrm{~Hz}$. It can be observed that the grid current suffers from a significant distortion and that the transient dc-link voltage dip increases to $10.7 \mathrm{~V}$. In addition, the total harmonic distortion (THD) in the grid current is also higher than that of the previous case in the steady state. Further, by using the windowed-FFT based RCG scheme, selective harmonic compensation can be achieved, which is preferred in case of a limited capacity of the compensators.

\section{CONCLUSIONS}

Control strategies for cascaded H-bridge multilevel active power filters (APFs) are presented in this paper, which include a current tracking controller, a dc-link voltage balancing controller and the reference current generation algorithm. The current controller is realized using a proportional- resonant (PR) regulator, which achieves zero steady-state error at selected harmonic frequencies. The power balancing mechanism of the dc-link capacitor voltages is analyzed and a voltage balancing controller (VBC) is presented. The Hanning Windowed-FFT algorithm is proposed for reference current generation (RCG) to mitigate the picket-fence effect of the conventional FFT under asynchronous sampling conditions. By using the Hanning Windowed-FFT algorithm and a PR current regulator, selective compensation is achieved. The effectiveness of the proposed algorithms for CHB-APFs is validated by simulation and experimental results.

\section{ACKNOWLEDGEMENT}

This project is supported by the Fundamental Research Funds for the Central Universities of China (ZYGX2011J093).

\section{REFERENCES}

[1] Y. Pal, A. Swarup, and Bhim Singh, "Control strategy for selective compensation of power quality problems through three-phase four-wire UPQC," Journal of Power Electronics, Vol. 11, No. 4, pp. 576-582, Jul. 2011.

[2] G. P. Zhang and L. Jinjun, "Analysis and specifications of switching frequency in parallel active power filters regarding compensation characteristics," Journal of Power Electronics, Vol. 10, No. 6, pp. 749-761, Nov. 2010.

[3] S. Zhang, K. Dai, B. Xie, and Y. Kang, "Parallel control of shunt active power filters in capacity proportion frequency allocation model," Journal of Power Electronics, Vol. 10, No. 4, pp. 419-427, Jul. 2010.

[4] J.-H. Lee, J.-K. Jeong, B.-M. Han, and B. Y. Bae, "New reference generation for a single- phase active power filter to improve steady state performance," Journal of Power Electronics, Vol. 10, No. 4, pp. 412-418, Jul. 2010.

[5] P. Flores, J. Dixon, M. Ortuzar, R. Carmi, P. Barriuso, and L. Moran, "Static Var compensator and active power filter with power injection capability, using 27-level inverters and photovoltaic cells," IEEE Trans. Ind. Electron. Vol. 56, No. 1, pp. 130-138, Jan. 2009.

[6] F. Defaÿ, A. Llor, and M. Fadel, "A predictive control with flying capacitor balancing of a multicell active power filter," IEEE Trans. Ind. Electron., Vol. 55, No. 9, pp. 3212-3220, Sep. 2008.

[7] Y. He, J. Liu, J Tang, Z. Wang, and Y. Zou, "Deadbeat control with a repetitive predictor for three-level active power filters," Journal of Power Electronics, Vol. 11, No. 4, pp. 583-590, Jul. 2011.

[8] A. M. Massoud, S. J. Finney, A. J. Cruden, B. W. Williams, "Three-phase, three-wire, five-level cascaded shunt active filter for power conditioning, using two different space vector modulation techniques," IEEE Trans. 
Power Del., Vol. 22, No. 4, pp. 2349-2361, Oct. 2007.

[9] Y.-M. Park, H.-S. Ryu, H.-W. Lee, M.-G. Jung, S.-H. Lee, "Design of a cascaded H-bridge multilevel inverter based on power electronics building blocks and control for high performance," Journal of Power Electronics, Vol. 10, No. 3, pp. 262-269, May 2010.

[10] E. Babaei, "Optimal topologies for cascaded submultilevel converters," Journal of Power Electronics, Vol. 10, No. 3, pp. 251-261, May 2010.

[11] H. Xue and R. Yang, "Optimal interpolating windowed discrete Fourier transform algorithms for harmonic analysis in power systems," IEE Proc.-Generation Transmission and Distribution, Vol. 150, No. 5, pp. 583-587, Sep. 2003.

[12] A.Testa, et. al., "On the processing of harmonics and interharmonics using hanning window in stardard framework," IEEE Transactions on Power Delivery, vol.19, No.1, pp.28-34, January 2004.

[13] V. Khadkikar, A Chandra, and B. N. Singh, "Generalized single-phase $\mathrm{p}-\mathrm{q}$ theory for active power filtering: simulation and DSP-based experimental investigation," IET Power Electronics, Vol. 2, No. 1, pp. 67-78, Jan. 2009.

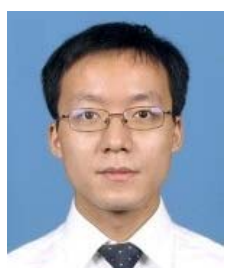

Yang Han was born in Chengdu, China, in 1982. He received his Ph.D. in Electrical Engineering from Shanghai Jiaotong University (SJTU), Shanghai, P. R. China, in 2010. He is a Member of the IEEE, the IEEE Industrial Electronics Society and the IEEE Power Electronics Society. He is a Senior Member of the China Power Supply Society (CPSS). He served as a member of the Organizing Committee of the 2011 International Conference on Electric Information and Control Engineering (ICEICE). He is an active Reviewer for the IEEE Transactions on Industrial Electronics, the IEEE Transactions on Power Electronics, etc. 